\title{
BMJ Open Published models that predict hospital readmission: a critical appraisal
}

\author{
Lisa Grossman Liu (D) ,' James R Rogers, ${ }^{1}$ Rollin Reeder, ${ }^{2,3}$ Colin G Walsh, ${ }^{2,3,4}$ \\ Devan Kansagara, ${ }^{5}$ David K Vawdrey, ${ }^{1,6}$ Hojjat Salmasian (D) ${ }^{7,8,9}$
}

To cite: Grossman Liu L, Rogers JR, Reeder R, et al. Published models that predict hospital readmission: a critical appraisal. BMJ Open 2021;11:e044964. doi:10.1136/ bmjopen-2020-044964

- Prepublication history and additional supplemental material for this paper are available online. To view these files, please visit the journal online (http://dx.doi.org/10.1136/ bmjopen-2020-044964).

Received 17 September 2020 Accepted 09 July 2021

Check for updates

(C) Author(s) (or their employer(s)) 2021. Re-use permitted under CC BY-NC. No commercial re-use. See rights and permissions. Published by BMJ.

For numbered affiliations see end of article.

Correspondence to

Dr Lisa Grossman Liu;

Ivg2104@cumc.columbia.edu

\section{ABSTRACT}

Introduction The number of readmission risk prediction models available has increased rapidly, and these models are used extensively for health decision-making. Unfortunately, readmission models can be subject to flaws in their development and validation, as well as limitations in their clinical usefulness.

Objective To critically appraise readmission models in the published literature using Delphi-based recommendations for their development and validation.

Methods We used the modified Delphi process to create Critical Appraisal of Models that Predict Readmission (CAMPR), which lists expert recommendations focused on development and validation of readmission models. Guided by CAMPR, two researchers independently appraised published readmission models in two recent systematic reviews and concurrently extracted data to generate reference lists of eligibility criteria and risk factors.

Results We found that published models $(n=81)$ followed 6.8 recommendations (45\%) on average. Many models had weaknesses in their development, including failure to internally validate $(12 \%)$, failure to account for readmission at other institutions $(93 \%)$, failure to account for missing data $(68 \%)$, failure to discuss data preprocessing $(67 \%)$ and failure to state the model's eligibility criteria (33\%). Conclusions The high prevalence of weaknesses in model development identified in the published literature is concerning, as these weaknesses are known to compromise predictive validity. CAMPR may support researchers, clinicians and administrators to identify and prevent future weaknesses in model development.

\section{INTRODUCTION}

In 2013, the Centers for Medicare and Medicaid Services (CMS) Hospital Readmission Reduction Program (HRRP) began to financially penalise US hospitals with excessive 30-day readmission rates, with the goal of improving patient care. Subsequently, research on readmission risk prediction models increased exponentially, ${ }^{2}$ with two distinct goals: (1) to identify high-risk patients for targeted interventions, and (2) to standardise institutions' readmission rates for use as a performance indicator. Preventable hospital readmissions cost CMS \$17 billion each year, ${ }^{3}$ and CMS penalties for subpar readmission rates totalled $\$ 566$ million in

\section{Strengths and limitations of this study}

This study appraises readmission models, of which dozens are published and which have limited regulatory oversight, to ensure high quality and usefulness for health decision-making.

- Recommendations are specific to readmission models and were developed using a modified Delphi process. Critical appraisal was undertaken independently by two researchers.

- Recommendations are limited to model development and validation, and future revision will be necessary as the field advances and developers adopt more modern techniques.

2018. ${ }^{4}$ Readmissions have received considerable attention due to this financial burden, their impact on patient care and their use as a performance indicator. ${ }^{56}$ Consequently, efforts to research ${ }^{78}$ and market $^{9}{ }^{10}$ readmission models have increased rapidly, and these models are used extensively for health decision-making.

However, uncritically accepting the results of any single model has risks. These models can be subject to flaws in their development and validation, as well as limitations in their clinical usefulness. Given the hundreds of readmission models now available, distinguishing the highest quality, most clinically useful models can be challenging for clinicians, researchers and healthcare administrators. In this study, we address this gap for readmission models in the published literature by critically appraising them. To conduct the critical appraisal, we developed Critical Appraisal of Models that Predict Readmission (CAMPR), which lists 15 Delphi-based expert recommendations for high-quality, clinically useful readmission models. CAMPR focuses on the unique considerations of readmission modelling, such as purpose, competing risks, outcome timing, risk factor definitions, data sources and thresholding. This manuscript discusses the expert recommendations and subsequent critical appraisal in detail, and 
provides reference lists of eligibility criteria and risk factors to consider when assessing readmission models.

\section{METHODS}

The Delphi method is a well-established structured communication technique for systematically seeking expert opinion on a specific topic. ${ }^{11-13}$ Traditionally, the first round uses open-ended questions to generate ideas from participants, whereas subsequent rounds rely on more structured communication to achieve consensus. In this study, we conducted two rounds of online expert surveys, the first open-ended and the second semistructured, and a third round consisting of expert application to the published literature. ${ }^{11}$

\section{Round 1: development of CAMPR (open-ended survey) Survey content}

A Delphi expert used an iterative process to develop the initial survey in collaboration with four physician experts in readmission models. The survey collected personal information on the respondents' institution(s) and relevant expertise, as well as information on models at the respondents' institution(s). Then, the survey assessed perceived barriers to model development and implementation, as well as strategies to overcome barriers and recommendations to improve models. The complete survey is available as online supplemental appendix A.

\section{Data collection}

To ensure rapidity and anonymity, provide convenience and recruit individuals from diverse backgrounds and geographical locations, we invited experts to participate electronically using Qualtrics Survey Software. Expert panels for Delphi studies traditionally have 12-20 members. ${ }^{14}$ Electronic participation enabled us to include more than 20 members, as desired due to the complex nature of the problem and the probable diversity of opinions. We distributed our survey via personalised, individual emails to all corresponding authors of readmission prediction studies from two recent systematic reviews $(2011,2016) .{ }^{12}$ Additionally, we publicly distributed it to members of the American Medical Informatics Association.

\section{Eligibility criteria}

We included both model developers and implementers in our expert panel to capture a broad range of perspectives on the readmission prediction literature. We required that participants speak English and self-report involvement in (1) the development of one or more readmission models, or (2) the implementation of one or more readmission models at one or more institutions.

\section{Data analysis}

Two researchers conducted thematic analysis in NVivo V.11 (QSR International). First, the researchers independently read each response and defined codes in a dictionary for the remaining analysis. Then, the

\begin{tabular}{|c|c|c|}
\hline Dimension & Key domain & Description of barrier \\
\hline \multirow[t]{4}{*}{ Development } & Validation & $\begin{array}{l}\text { Inadequate validation } \\
\text { causing poor model } \\
\text { discrimination when } \\
\text { generalised }\end{array}$ \\
\hline & Features & $\begin{array}{l}\text { Over-reliance on biomedical } \\
\text { features from administrative } \\
\text { and claims data }\end{array}$ \\
\hline & Timeframe & $\begin{array}{l}\text { Possibility that the } 30 \text {-day } \\
\text { timeframe is not optimal for } \\
\text { accurate prediction }\end{array}$ \\
\hline & Data access & $\begin{array}{l}\text { Dependence on } \\
\text { inaccessible, low quality, } \\
\text { outdated or manually } \\
\text { entered data }\end{array}$ \\
\hline \multirow[t]{5}{*}{ Implementation } & Resources & $\begin{array}{l}\text { Insufficient personnel, } \\
\text { statistical expertise or } \\
\text { financial resources }\end{array}$ \\
\hline & Vision & $\begin{array}{l}\text { Lack of leadership, purpose } \\
\text { or policy priorities related to } \\
\text { readmission prediction }\end{array}$ \\
\hline & $\begin{array}{l}\text { Clinical } \\
\text { relevance }\end{array}$ & $\begin{array}{l}\text { Unclear clinical utility, } \\
\text { actionability or relevance }\end{array}$ \\
\hline & $\begin{array}{l}\text { Workflow } \\
\text { integration }\end{array}$ & $\begin{array}{l}\text { Poor integration into the } \\
\text { clinical workflow }\end{array}$ \\
\hline & Maintenance & $\begin{array}{l}\text { Inadequate maintenance or } \\
\text { continuous improvement }\end{array}$ \\
\hline
\end{tabular}

researchers independently coded all responses using codes corresponding to the dictionary and summarised themes that emerged. Together, the researchers reviewed and named common themes that emerged, and resolved conflicts by discussion. To enhance confirmability, we shared summaries of coded data with three participants and asked for their confirmation or revisions to interpretation.

\section{Round 2: further development of CAMPR (semistructured survey)}

Preliminary version

Based on the thematic analysis in round 1, the study team identified 48 preliminary recommendations to operationalise two quality dimensions of readmission models: (1) development and (2) implementation. Each preliminary recommendation addressed one of four key thematic domains for development and five key thematic domains for implementation, identified via expert consensus (table 1).

\section{Survey content and data collection}

For each preliminary recommendation, the second survey asked participants to score the usefulness and content validity. Free-text fields enabled participants to add additional comments on each individual recommendation, as well as CAMPR in its entirety. The study team 
reviewed the survey before electronic delivery using Qualtrics. We distributed the survey via personalised emails to all previous eligible respondents who had agreed to additional contact. The complete survey is available as online supplemental appendix B.

\section{Data analysis}

We conducted a quantitative descriptive analysis of usefulness and content validity using R-3.3.3. Preliminary recommendations with a usefulness and content validity below predetermined thresholds $(<50 \%$ useful or valid) were excluded, unless the free-text commentary indicated that the usefulness and content validity would greatly improve with revision. The study team reviewed all freetext commentary and refined, reworded or combined recommendations accordingly.

\section{Round 3: application of CAMPR}

Modified preliminary version

After refinement and reduction in round 2, the modified preliminary version contained 34 recommendations. We identified 23 development-related recommendations for inclusion in CAMPR, which primarily reflect the four key development domains identified in round 1 (validation, features, timeframe, data access). The remaining 11 implementation-related recommendations, which primarily reflect the five key implementation domains (resources, vision, clinical relevance, workflow integration and maintenance), will be reported on separately.

\section{Iterative validation}

Two researchers applied CAMPR to all published readmission prediction models identified in all known systematic reviews $(2011,2016) .{ }^{12}$ First, the researchers independently applied CAMPR to one-third of studies, then revised it to improve clarity, resolve discrepancies in application and combine redundant recommendations, which reduced the total number to 15 . Then, the researchers independently applied the finalised version to all studies (detailed in online supplemental appendix C) and assessed inter-rater reliability. Conflicts were resolved by discussion.

\section{Data extraction}

Manual data extraction occurred concurrently with the application of CAMPR, to better assess characteristics of included studies. Importantly, we extracted eligibility criteria and risk factors for each readmission model, to generate reference lists for future developers (available tables 2 and 3 in the Results section). Examples of other types of extracted data include the readmission timeframe used (relevant to recommendation \#6) and the validation technique used (relevant to recommendation \#13). Two researchers developed the data extraction tool based on the initial review of one-third of studies. One researcher extracted data from each study, and another reviewed all extractions for completeness and accuracy.

\section{Data Analysis}

All analyses were performed in R V.3.6.3. We used Cohen's kappa and percentage agreement to measure inter-rater reliability. We stratified literature into recent (2011-2015) and early (1985-2010), using the year of CMS HRRP (2011) as our cut-off. We conducted bivariate analyses to assess whether adherence to each recommendation differed between recent and early literature, using an unequal variances t-test. Furthermore, we used Spearman's rank correlation to examine whether overall adherence to recommendations differed by publication year. When classifying risk factors, we used the same classification as the first systematic review, ${ }^{1}$ with the added category 'institution-related' as suggested by expert consensus.

\section{RESULTS \\ Development of CAMPR \\ Round 1}

We successfully contacted 75 out of 81 corresponding authors who developed unique readmission models published from 1985 to 2015, of whom 14 (19\%) completed our survey. An additional 49 respondents completed our survey after we publicly distributed it, of which we included 40 who had experience implementing readmission models. The final 54 eligible experts (14 developers and 40 implementers, characterised in online supplemental appendix D) represented 20 unique models, including well-known models such as LACE and CMS-endorsed models. Of 14 developers, only 7 (50\%) reported that any institution currently used their model in any capacity. Table 4 reports expert-identified barriers to developing, validating and implementing readmission models, as well as strategies to overcome barriers.

\section{Rounds 2 and 3}

We had permission to reconnect with 22 previous respondents, of whom 5 (23\%) completed our second survey.

\section{Application of CAMPR}

We included 81 published readmission models in our critical appraisal. ${ }^{15-95}$ We found that published models followed 6.8 out of 15 recommendations (45\%) on average. Fifty-five out of $81(68 \%)$ followed less than half the recommendations, and no study followed every recommendation, suggesting an opportunity for improvement. Table 5 presents the percentages of published readmission models following each recommendation, stratified by publication year. Models published recently (2011-2015, $\mathrm{n}=55,68 \%)$ followed significantly more recommendations than models published earlier (1985$2010, \mathrm{n}=26,32 \%)(7.1$ vs $6.1, \mathrm{p}=0.03)$, and publication year weakly correlated with recommendations followed $(\rho=0.27, p=0.02)$, suggesting slight improvement in model quality over time as the field developed. Model types included regression $(77,95 \%)$, random forest (3, $4 \%)$, neural network $(3,4 \%)$, decision tree $(2,2 \%)$, discriminant analysis $(2,2 \%)$, support vector machine 
Table 2 Barriers to developing and implementing readmission models

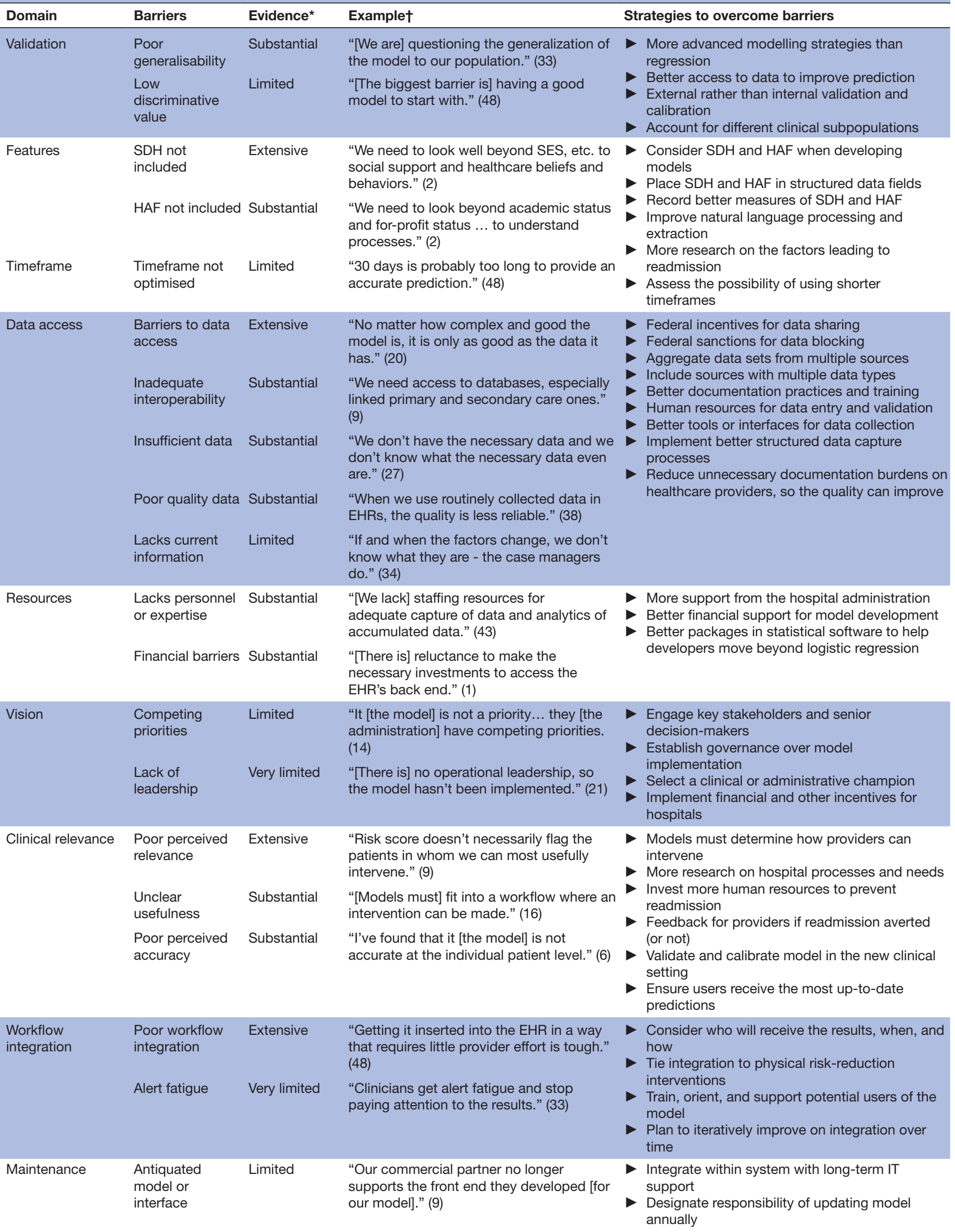


Table 2 Continued

Domain Barriers $\quad$ Evidence* $^{*} \quad$ Example† Strategies to overcome barriers

*Extensive evidence ( $\geq 8$ mentions); substantial evidence (4-7 mentions); limited evidence (2-3 mentions), very limited evidence (1 mention). †Numbers in parentheses refer to participant study ID.

EHR, electronic health record; HAF, healthcare-associated factors; IT, information technology; SDH, social determinants of health; SES, socioeconomic status.

$(1,1 \%)$ and unclear $(1,1 \%)$. We found moderate-tohigh inter-rater reliability for applying CAMPR (Cohen's kappa $=0.76$, agreement $=88 \%$ ). Here, we summarise each recommendation in CAMPR and present the critical appraisal results. Additional results are in online supplemental appendix D. The complete dataset is available on request. CAMPR is available as online supplemental appendix E.
Recommendation \#1: is the model's purpose and eligibility criteria explicitly stated?

\section{About the recommendation}

Readmission models traditionally serve one of two purposes, or intended applications: (1) to identify patient candidates for targeted interventions to prevent readmission, or (2) to risk-adjust readmission rates for hospital quality comparison. ${ }^{1}$ Developers should clearly state

Table 3 Critical Appraisal of Models that Predict Readmission (CAMPR)

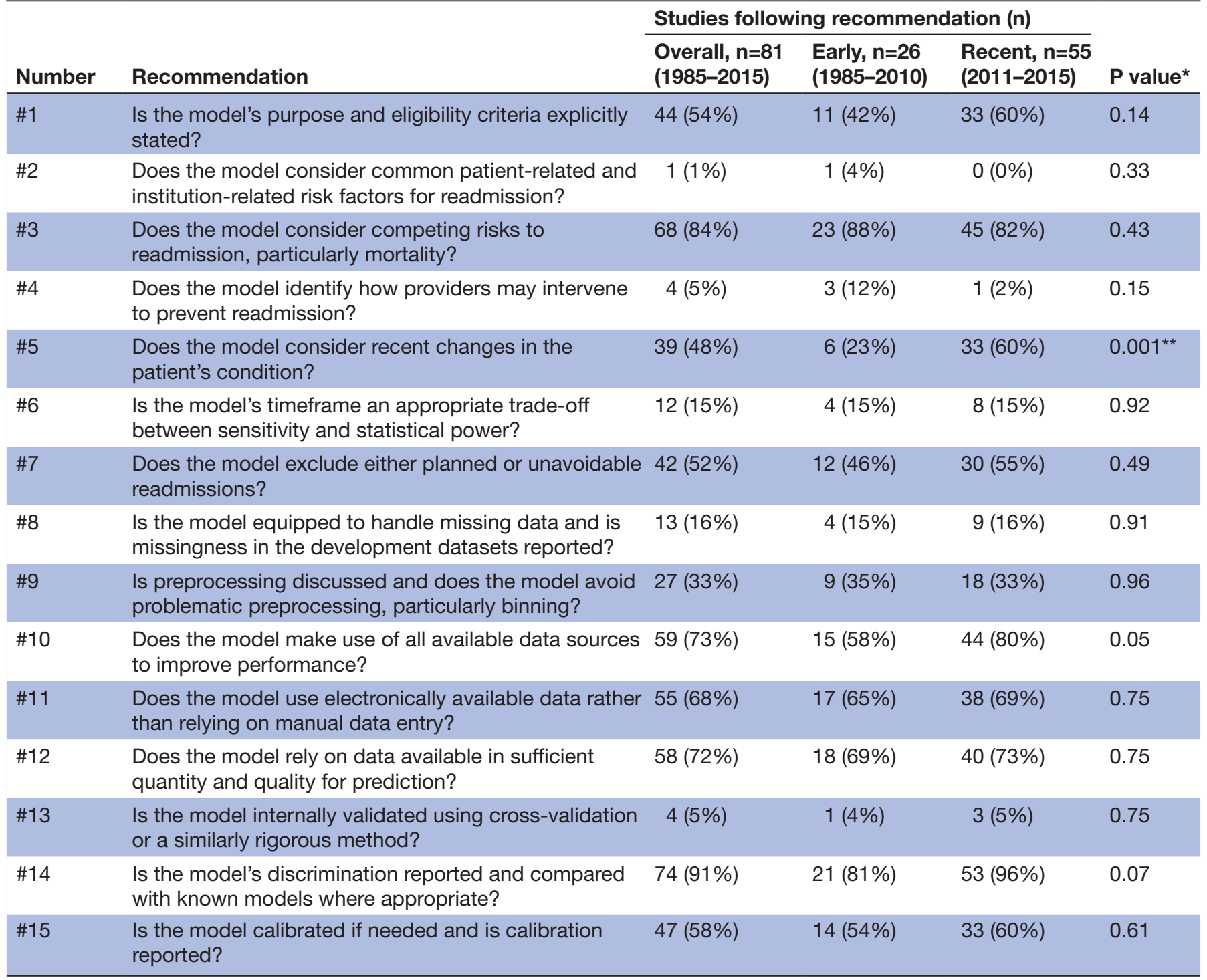

*Significant at $\mathrm{p}=0.05 ;{ }^{* *}$ significant at $\mathrm{p}=0.001$.

${ }^{*}$ Comparison is between models published earlier (1985-2010) and more recently (2011-2015). 
Table 4 Eligibility criteria for readmission prediction models*

\section{Criterion}

Studies (n, \%)

Citations (see online supplemental appendix E)

Inclusion criteria

$\begin{array}{lcl}\text { Age } & 48(59 \%) & \\ >18 \text { years (adults only) } & 25(31 \%) & 374043454749-51555665676873747880-82858688929395 \\ >65 \text { years (elderly only) } & 19(23 \%) & 17-192125272832-34384852606676777990 \\ \text { Other } & 5(6 \%) & 2336426293 \\ \text { Condition specific } & 45(56 \%) & 1821262933464767-6971-7586 \\ \text { Heart failure } & 16(20 \%) & 346-5864-6670 \\ \text { AMl or other cardiovascular } & 11(14 \%) & 194647627677 \\ \text { Pneumonia or other pulmonary } & 6(7 \%) & 232428315463 \\ \text { Multiple } & 6(7 \%) & 8084-93 \\ \text { Other } & 11(14 \%) & 35363840444578-8387 \\ \text { Service specific } & 24(30 \%) & 57606389-92 \\ \text { Medicine } & 14(17 \%) & 529495 \\ \text { Surgery } & 7(9 \%) & \\ \text { Other } & 3(4 \%) & 1517-192125283233414858606690 \\ \text { Beneficiaries } & 22(27 \%) & 22363839535861 \\ \text { Medicare } & 15(19 \%) & 7(9 \%) \\ \text { Veterans } & \end{array}$

Exclusion criteria

Disposition $28(35 \%)$

Left against medical advice $11(14 \%)$

Skilled nursing or other care facility $10(12 \%)$

Hospice care $8(10 \%)$

Different healthcare system $4(5 \%)$

2140424546505860618082

32374350566168788693

Transfers 26 (32\%)

Transfer-out (to other institution or service) 24 (30\%)

Transfer-in (from other institution or service) $3(4 \%)$

3243496178858688

Service or condition $24(30 \%)$

Rehabilitation $10(12 \%)$

Psychiatric $8(10 \%)$

Obstetric/neonatal $8(10 \%)$

Transplant

$4(5 \%)$

Other (ESRD, trauma, etc)

$5(6 \%)$

35364494

Length of stay

$<24$ hours (same-day discharge or $\quad 16(20 \%)$

procedure)

$>30$ days $\quad 5(6 \%)$

$17-22273340424546565861-6368727476778093$

475361

Hospitalisation type $9(11 \%)$

Planned (aka elective) $3(4 \%)$

Non-acute (aka non-emergent) $3(4 \%)$

Observation only $2(2 \%)$

$7(9 \%)$

Study related

Unavailable for follow-up (no telephone, $\quad 5$ (6\%)

$214349-51565861-6368727476778093$

2227435051555661

202342434751555661

$7584-86$

etc)

Cannot consent (poor cognition) $2(2 \%)$

Continued 
Table 4 Continued

\begin{tabular}{lcl}
\hline Criterion & Studies (n, \%) & Citations (see online supplemental appendix E) \\
\hline Non-English speaking & $2(2 \%)$ & 6773 \\
$\begin{array}{l}\text { Non-residents (outside the primary service } \\
\text { area) }\end{array}$ & $7(9 \%)$ & 20333646656793 \\
$\begin{array}{l}\text { No insurance coverage (for part or all of the } \\
\text { data extraction period) }\end{array}$ & $5(6 \%)$ & 4256606677 \\
$\begin{array}{l}\text { Data-related (not recent or validated) } \\
\text { Readmitted for different condition }\end{array}$ & $3(4 \%)$ & 32352 \\
\hline
\end{tabular}

An extended version of this table is available in online supplemental appendix D.

*This table does not cover exclusions based on mortality (see recommendation \#3) or missingness (see recommendation \#8).

AMI, acute myocardial infarction; ESRD, end-stage renal disease.

which purpose their model serves, one or both. Developers should also define the target population by specifying eligibility criteria for patient inclusion in model development. Specifying eligibility criteria is critical to ensure implementers understand when each model applies, as unjustified application is a major reason why predictions fail. ${ }^{96} 97$

\section{Critical appraisal results}

Eighteen out of 81 studies (22\%) did not define their model's purpose. Of the remaining models, 46 (57\%) were for preventing readmission, $15(19 \%)$ were for hospital quality comparison and $2(2 \%)$ were for both. Table 4 provides an abbreviated reference list of eligibility criteria for published readmission models (the full reference list is available in online supplemental appendix D). Twenty-seven models (33\%) did not specify their eligibility criteria.

Recommendation \#2: does the model consider common patientrelated and institution-related risk factors for readmission?

\section{About the recommendation}

Developers should show that they considered risk factors or features that were included in previous models. Notably, institution-related factors such as hospital name should not be used in models for hospital quality comparison, as they can mask differences in hospital quality.

\section{Critical appraisal results}

Table 5 provides an abbreviated reference list of known risk factors for readmission and their frequency of inclusion in published models (the full reference list is available as online supplemental appendix D). Based on expert consensus and the existing literature, we identified seven categories of factors. ${ }^{1}$ Categories included (1) demographics (included in 75 models or 93\%), (2) disease related $(80,99 \%)$, (3) functional ability (21, $26 \%)$, (4) healthcare utilisation $(66,81 \%)$, (5) medication related $(33,41 \%),(6)$ social determinants of health $(53,65 \%),(7)$ institution related $(16,23 \%)$. Five studies (out of 15, 33\%) mistakenly used institution-related risk factors in models for hospital quality comparison.
Recommendation \#3: does the model consider competing risks to readmission, particularly mortality?

About the recommendation

Death is a competing risk to readmission and may substantially impact readmission prediction depending on the target population. ${ }^{636768} \mathrm{~A}$ high mortality rate may reduce model discrimination because death and readmission share similar predictive features. Ignoring mortality may limit insight about risk factors, and unaccounted changes in mortality may cause model drift. Developers should indicate that they accounted for both in-hospital and post-discharge mortality, as well as other competing risks to readmission (eg, transfers) ${ }^{28}$

\section{Critical appraisal results}

Thirteen models (16\%) did not account for mortality, 40 (49\%) accounted for in-hospital mortality only, $5(6 \%)$ accounted for post-discharge mortality only and 21 (26\%) accounted for both.

Recommendation \#4: does the model identify how providers may intervene to prevent readmission?

\section{About the recommendation}

The expert group recognised that building actionable models, which identify where providers can intervene on risk factors to prevent readmissions, is critical to clinical usefulness. An actionable model may (1) identify modifiable risk factors on the individual level, ${ }^{36} 6290$ or (2) identify which individuals will benefit most from intervention, which may not coincide with readmission risk. Notably, non-modifiable risk factors like age can obscure modifiable ones like polypharmacy or quality of care $^{6098}{ }^{99}$; therefore, managing collinearity ${ }^{100}$ is important. In the future, predicting benefit will become easier as options for intervention become more well researched. ${ }^{101}$

\section{Critical appraisal results}

Four published models (5\%) identified modifiable factors on the individual level. No models have predicted which individuals would benefit most from intervention. 
Table 5 Risk factors included in readmission prediction models

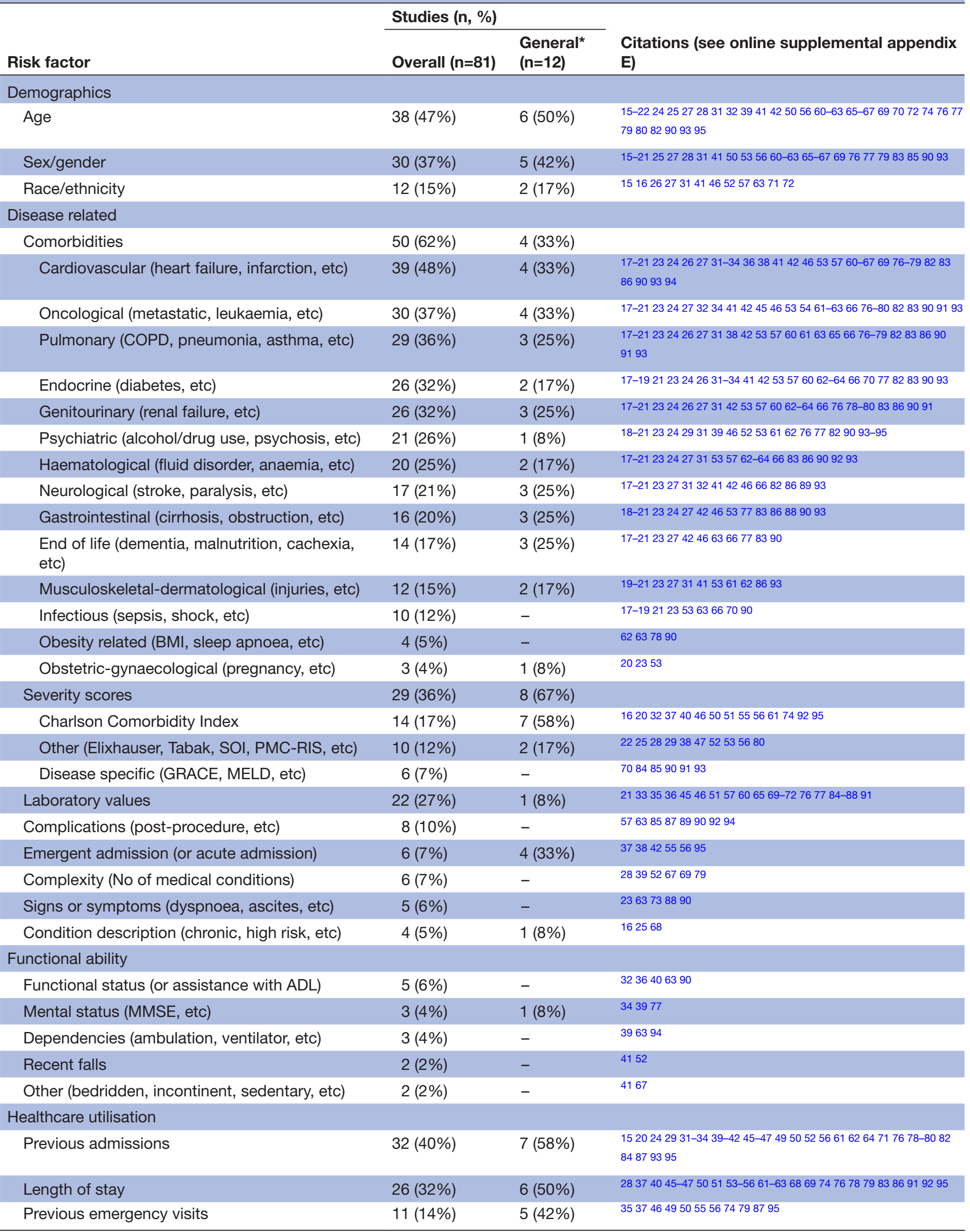


Table 5 Continued

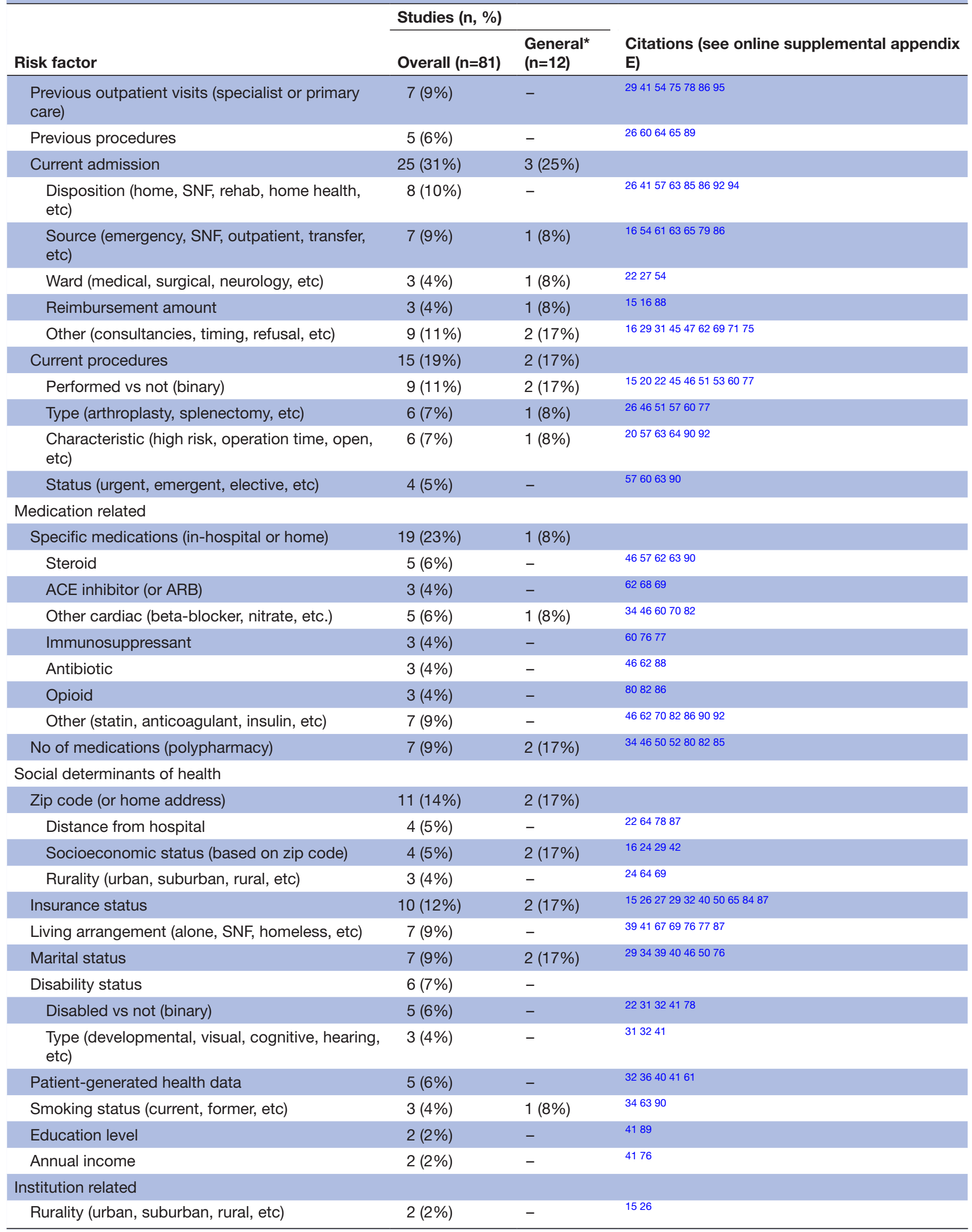




\begin{tabular}{llll}
\hline & \multicolumn{2}{c}{ Studies (n, \%) } & \\
\cline { 2 - 3 } Risk factor & Overall $(\mathbf{n = 8 1 )}$ & $\begin{array}{l}\text { General } \\
(\mathbf{n = 1 2})\end{array}$ & $\begin{array}{l}\text { Citations (see online supplemental appendix } \\
\text { E) }\end{array}$ \\
\hline Standardised admission ratios & $2(2 \%)$ & $1(8 \%)$ & 1631 \\
\hline Identification code & $2(2 \%)$ & $1(8 \%)$ & 4252 \\
\hline
\end{tabular}

We could not extract factors from seven studies, either due to poor reporting or lack of feature selection. ${ }^{30434448585981}$ An extended version of table is available in online supplemental appendix $D$.

*'General' indicates models for the general hospitalised population, and is not restricted to any one target population. ${ }^{1620273437424749-515556}$

ACE, Angiotensin-converting enzyme; ADL, activities of daily living; ARB, Angiotensin receptor blocker; BMI, body mass index; COPD, chronic obstructive pulmonary disease; DNR, do not resuscitate; MMSE, Mini Mental State Exam; SNF, skilled nursing facility; SOI, severity of illness.

Recommendation \#5: does the model consider recent changes in the patient's condition?

\section{About the recommendation}

A model that does not account for recent changes in the patient's condition may give an outdated prediction, limiting its clinical usefulness and eroding trust in its predictions. The expert group recommended that models that give predictions near hospital discharge (ie, most current models) should account for changes during hospitalisation, including treatment effects, hospitalacquired conditions and social support status.

\section{Critical appraisal results}

Thirty-nine models (48\%) accounted for changes during hospitalisation.

Recommendation \#6: is the model's timeframe an appropriate trade-off between sensitivity and statistical power?

\section{About the recommendation}

Researchers initially selected the 30-day timeframe as the optimal trade-off between statistical power and likelihood of association with the index admission..$^{28}$ As common data models and health information exchange support larger datasets for model development, shorter timeframes such as 7 days may enable greater sensitivity for readmissions associated with the index admission without loss in statistical power. ${ }^{102}$ Therefore, developers should consider assessing prediction accuracy using multiple timeframes, as relevant to the clinical context and dataset size, to determine the best trade-off between sensitivity and statistical power. Timeframes should begin at discharge (as standardised by $\mathrm{CMS})^{103}$ to prevent immortal persontime bias. $^{63}$

\section{Critical appraisal results}

Sixty-three models $(78 \%)$ used the standardised 30-day timeframe adopted by CMS, while $2(2 \%)$ used 7 days, 3 (4\%) 28 days, 5 (6\%) 60 days and $9(11 \%) 1$ year. Twelve studies $(15 \%)$ considered more than one timeframe, of which $7(9 \%)$ modelled readmission risk using hazard rates. Nine studies $(11 \%)$ inappropriately defined timeframes as beginning at admission, rather than discharge, and $14(17 \%)$ did not specify when their timeframe began.

Recommendation \#7: does the model exclude either planned or unavoidable readmissions?

\section{About the recommendation}

Planned readmission is defined as non-acute readmission for scheduled procedures. Planned readmissions should be excluded, as consistent with the standardised definition of all-cause readmission. ${ }^{17-19} 66$ Unavoidable readmission is defined more broadly as readmission not preventable through clinical intervention. ${ }^{28}$ As researchers develop standardised algorithms to more effectively identify unavoidable readmissions, ${ }^{4761} 104$ using the broader definition may enable greater sensitivity and improve the relevance of predictions to the clinical setting. Therefore, developers should consider excluding unavoidable readmissions if it is useful, such as in multiple sclerosis, where the disease inevitably progresses and later readmissions become increasingly unavoidable. Notably, exclusion criteria can be highly complex and require third-party processing (eg, Vizient). Ideally, developers should publish their code. If not, the readmission outcome should be sufficiently defined to ensure transparency and reproducibility. ${ }^{105}$

\section{Critical appraisal results}

Thirty-nine models (48\%) did not explicitly exclude planned readmissions. The remaining models either excluded planned readmissions $(38,47 \%)$ or excluded unavoidable readmissions more broadly $(4,5 \%)$.

Recommendation \#8: is the model equipped to handle missing data and is missingness in the development datasets reported? About the recommendation

Developers should explicitly state whether their model handles missingness and how, such as designating a 'missing' category for categorical variables, or multiple regression imputation for continuous variables. Dropping individuals with excess missingness is problematic because it decreases models' generalisability to future individuals with excess missingness and falsely increases 
model performance in cases of structural missingness. Developers should also report on missingness in the datasets used for model development, so that implementers can determine potential generalisability to their realworld datasets.

\section{Critical appraisal results}

Only 34 studies (42\%) discussed how their model handled missingness. Of these, 20 (25\%) used one or more inappropriate techniques, including (1) dropping individuals with excess missingness $(17,21 \%)$, and (2) binning or imputation which was done improperly $(3,4 \%)$.

Recommendation \#9: is preprocessing discussed and does the model avoid problematic preprocessing, particularly binning?

\section{About the recommendation}

Developers should explain their data preprocessing methods, because problematic methods may produce models with less-than-optimal predictive performance. ${ }^{96}$ One example of a problematic method is binning. ${ }^{106}$ Originally intended to improve interpretability, binning can cause information loss, and is no longer justifiable given users' need for accurate predictions and modern interpretability techniques. In particular, manual or arbitrary binning, without clustering or splines, may decrease performance and introduce noise. ${ }^{107}$

\section{Critical appraisal results}

Only 27 studies (33\%) discussed one or more data preprocessing techniques, despite mostly using regression models, which can be highly sensitive to small changes. Commonly discussed techniques included binning, interaction terms and transformations to resolve skewness, non-linearity and outliers.

\section{Recommendation \#10: does the model make use of all available} data sources to improve performance?

\section{About the recommendation}

Developers should make use of publicly available data sources where possible and appropriate to the model's purpose, such as the Social Security Death Index to determine post-discharge mortality (see recommendation \#3) or curated public datasets to externally validate (see recommendation \#13). Other data sources such as health information exchanges can help assess readmission at multiple institutions, which is desirable to better estimate the true readmission rate. When considering data sources from multiple institutions, such as with health information exchanges, developers should account for hospitallevel patterns and clustering of readmission risk, which may occur because quality of care and data collection practices vary between institutions. ${ }^{36} 6168$

\section{Critical appraisal results}

In the literature, data sources included claims (19, 23\%), administrative datasets $(33,41 \%)$, electronic health records $(42,52 \%)$, disease-specific registries $(12,15 \%)$, research datasets $(11,14 \%)$, death registries $(9,11 \%)$, health information exchanges or linkages $(4,5 \%)$, and surveys or patient-generated health data $(3,4 \%)$. Seventyfive studies $(93 \%)$ assessed readmission at only one institution, likely underestimating the true readmission rate. Thirty-nine studies (48\%) used a single data source (administrative datasets: 15, 19\%; electronic health records: $17,21 \%$ ).

Recommendation \#11: does the model use electronically available data rather than relying on manual data entry? About the recommendation

Developers should incorporate risk factors that will be available electronically at the time of prediction and avoid manual data entry by providers or research assistants. Manual data entry may inhibit widespread implementation, by consuming human resources and preventing automated generation of predictions.

\section{Data extraction results}

Twenty-six models (32\%) relied on manual data entry.

Recommendation \#12: does the model rely on data available in sufficient quantity and quality for prediction?

\section{About the recommendation}

Developers should indicate whether data included in their model can be accessed in sufficient amounts and quality for development and implementation. 'Sufficient' is subjective and requires consideration of real-world missingness. Automated quality assurance, which identifies erroneous entries (eg, age $>120$ years) and incorrect data combinations (eg, former smoker YES, never smoker YES), may help to improve quality.

\section{Critical appraisal results}

Twenty-three studies (28\%) identified problems with either data quantity (17 out of $23,74 \%$ ) or quality ( 8 out of $23,26 \%$ ).

Recommendation \#13: is the model internally validated using cross-validation or a similarly rigorous method?

\section{About the recommendation}

The importance of using repeated k-fold cross-validation or a similarly rigorous method is well established. Splitsample validation is insufficient and may cause unstable and suboptimal predictive performance. ${ }^{108-110}$ If the model is intended for generalised use at more than one institution, developers or implementers should confirm external validity using one or more external, representative and independent datasets, from another institution or source. Internal validation alone is insufficient to ensure generalisability. ${ }^{108}$

\section{Critical appraisal results}

Ten models (12\%) were not validated at all, $64(79 \%)$ were internally validated only and $7(9 \%)$ were internally and externally validated. For internal validation, 46 (57\%) used random split-sample, 11 (14\%) used split-sample by time, 12 (15\%) used bootstrapping, 3 (4\%) used crossvalidation and $1(1 \%)$ used out-of-bag estimates. 
Recommendation \#14: is the model's discrimination reported and compared with known models where appropriate?

\section{About the recommendation}

It is commonly accepted practice to prominently and clearly report discrimination using appropriate and well-known measures beyond just the concordance (c) statistic. ${ }^{111}$ Where possible, comparison with an established baseline is essential, because so many models already exist. Developers should compare performance using statistical tests with cross-validation or another method, and only compare models with similar eligibility criteria.

\section{Critical appraisal results}

Seven models (8\%) did not report discrimination. Commonly reported measures included the c statistic (47, $58 \%)$, sensitivity or specificity $(23,28 \%)$, area under the receiver operating characteristic curve (19, 23\%), negative or positive predictive value $(18,22 \%)$, integrated discrimination improvement $(5,6 \%)$ and net reclassification improvement $(3,4 \%)$.

\section{Recommendation \#15: is the model calibrated if needed and is} calibration reported?

\section{About the recommendation}

Proper calibration is critical for sorting patients in descending order of readmission risk for making intervention decisions. It is commonly accepted practice to report calibration using calibration curves with no binning. ${ }^{112} 113$ Reporting the Hosmer-Lemeshow (HL) goodness-of-fit statistic is insufficient, as a non-significant HL statistic does not imply the model is well calibrated, and the HL statistic is often insufficient to detect quadratic overfitting effects common to support vector machines and treebased models. $^{112}$

\section{Critical appraisal results}

Thirty-seven models (44\%) did not assess calibration. Commonly reported measures included the HL statistic $(29,36 \%)$ and observed-to-expected ratios $(17,21 \%)$.

\section{DISCUSSION}

In this study, we critically appraised readmission models using 15 Delphi-based expert recommendations for development and validation. Interestingly, we found that many published readmission models did not follow the experts' recommendations. This included failure to internally validate $(12 \%)$, failure to account for readmission at other institutions $(93 \%)$, failure to account for missing data $(68 \%)$, failure to discuss data preprocessing $(67 \%)$ and failure to state the model's eligibility criteria (33\%). The high prevalence of these weaknesses in model development identified in the published literature is concerning, because these weaknesses are known to compromise predictive validity. Identification of weaknesses in these domains should undermine confidence in a model's predictions.
In our expert surveys, several lessons emerged, most notably about improving models' relevance to clinical care and integration of predictions into clinicians' workflows. In particular, experts expressed concern that models identified the highest-risk patients rather than the patients who might benefit most from intervention, which led to recommendation \#4. Experts also noted that the published literature in existing systematic reviews and therefore our critical appraisal is focused on development and internal validation. This suggests that literature on external validation and implementation is less common. Additional efforts to research external validation and implementation could improve readmission models, by making them more applicable to a broader patient population.

In the future, CAMPR may be a convenient teaching aid for model implementers and users at healthcare institutions, such as clinicians and healthcare administrators, as well as for model developers in academic and commercial research. CAMPR does not explain the detailed logic and methods of developing and implementing predictive models, and those looking for comprehensive advice should consult other resources. Finally, CAMPR is not intended as a reporting standard for academic studies, and responses to CAMPR recommendations should not be used to derive an overall score. An overall score may disguise critical weaknesses that should diminish confidence in model predictions. Rather than generating an overall score, consider the potential impact of failing to follow each recommendation, and how that may interact with the use of that model in the given patient population.

We developed CAMPR using a modified Delphi process consisting of two online rounds, which we found faster and more practical than conducting the traditional in-person meetings and three rounds. Beyond readmission modelling, other predictive modelling domains in healthcare (eg, sepsis risk, mortality risk, etc) could benefit from similar guidance. Thinking beyond better modelling techniques is essential, or model predictions will remain of limited clinical use. This includes thinking about how to generate better datasets, thinking about model drift and maintenance over time, ${ }^{114}$ and thinking about how to clinicians should act on predictions.

\section{Limitations}

The study used a modified Delphi process, which may lack rigour compared with the traditional Delphi process. We used an 'opt-in' process to recruit experts, and this selfselection bias may have led to missed recommendations or opinions. Fewer participants responded to the second round than expected, although the number was sufficient for the Delphi process. Future revision of recommendations will likely be necessary as the field advances and developers adopt more modern techniques. CAMPR is not intended as a reporting standard, and a more formal evaluation of construct validity and generalisability would be needed before it could be used as such. 
Author affiliations

${ }^{1}$ Department of Biomedical Informatics, Columbia University, New York, New York, USA

${ }^{2}$ Department of Biomedical Informatics, Vanderbilt University, Nashville, Tennessee, USA

${ }^{3}$ Department of Medicine, Vanderbilt University, Nashville, Tennessee, USA

${ }^{4}$ Department of Psychiatry, Vanderbilt University, Nashville, Tennessee, USA

${ }^{5}$ Department of Medicine, Oregon Health and Science University and VA Portland

Health Care System, Portland, Oregon, USA

${ }^{6}$ Steele Institute for Health Innovation, Geisinger, Danville, Pennsylvania, USA

${ }^{7}$ Division of General Internal Medicine, Brigham and Women's Hospital, Boston,

Massachusetts, USA

${ }^{8}$ Harvard Medical School, Boston, Massachusetts, USA

${ }^{9}$ Mass General Brigham, Somerville, Massachusetts, USA

\section{Twitter Lisa Grossman Liu @LisaGrossmanLiu}

Contributors HS conceptualised this work. LGL and RR conducted and analysed the expert surveys. HS, CGW, DK and DKV provided supervision throughout the survey process. LGL and JRR conducted the critical appraisal and data extraction, and LGL performed the associated analyses. LGL drafted the manuscript, and all authors contributed to refining all sections and critically editing the paper.

Funding This work was supported by the National Library of Medicine (F31LM054013, PI: LGL).

Competing interests None declared.

Patient consent for publication Not required.

Ethics approval The Columbia University Medical Center Institutional Review Board approved the studies (Protocol AAAR0148). We used online surveys to collect opinions about predictive models from expert researchers. In accordance with 45CFR46.101, the Columbia University Medical Center Institutional Review Board determined this to be a Category 2 exemption and did not require written informed consent.

Provenance and peer review Not commissioned; externally peer reviewed.

Data availability statement Data are available on reasonable request. Please email the corresponding author.

Supplemental material This content has been supplied by the author(s). It has not been vetted by BMJ Publishing Group Limited (BMJ) and may not have been peer-reviewed. Any opinions or recommendations discussed are solely those of the author(s) and are not endorsed by BMJ. BMJ disclaims all liability and responsibility arising from any reliance placed on the content. Where the content includes any translated material, BMJ does not warrant the accuracy and reliability of the translations (including but not limited to local regulations, clinical guidelines, terminology, drug names and drug dosages), and is not responsible for any error and/or omissions arising from translation and adaptation or otherwise.

Open access This is an open access article distributed in accordance with the Creative Commons Attribution Non Commercial (CC BY-NC 4.0) license, which permits others to distribute, remix, adapt, build upon this work non-commercially, and license their derivative works on different terms, provided the original work is properly cited, appropriate credit is given, any changes made indicated, and the use is non-commercial. See: http://creativecommons.org/licenses/by-nc/4.0/.

ORCID iDs

Lisa Grossman Liu http://orcid.org/0000-0002-1153-1590

Hojjat Salmasian http://orcid.org/0000-0002-9004-7149

\section{REFERENCES}

1 Kansagara D, Englander $\mathrm{H}$, Salanitro $\mathrm{A}$, et al. Risk prediction models for hospital readmission: a systematic review. JAMA 2011;306:1688-98.

2 Zhou H, Della PR, Roberts P, et al. Utility of models to predict 28-day or 30-day unplanned Hospital readmissions: an updated systematic review. BMJ Open 2016;6:e011060.

3 Agency for Healthcare Research and Quality. Statistical Brief \#172: Conditions With the Largest Number of Adult Hospital Readmissions by Payer, 2011, 2014. Available: https://www.hcupus.ahrq.gov/reports/statbriefs/sb172-Conditions-ReadmissionsPayer.pdf
4 Centers for Medicare \& Medicaid Services. Federal register volume 83 , number 160 , rules and regulations, 2018. Available: https:// www.govinfo.gov/content/pkg/FR-2018-08-17/html/2018-16766. $\mathrm{htm}$

5 Ody C, Msall L, Dafny LS, et al. Decreases in readmissions credited to Medicare's program to reduce hospital readmissions have been overstated. Health Aff 2019;38:36-43.

6 Wadhera RK, Joynt Maddox KE, Wasfy JH, et al. Association of the hospital readmissions reduction program with mortality among Medicare beneficiaries hospitalized for heart failure, acute myocardial infarction, and pneumonia. JAMA 2018;320:2542-52.

7 Lin Y-W, Zhou Y, Faghri F, et al. Analysis and prediction of unplanned intensive care unit readmission using recurrent neural networks with long short-term memory. PLoS One 2019;14:e0218942.

8 Maltenfort MG, Chen Y, Forrest CB. Prediction of 30-day pediatric unplanned hospitalizations using the Johns Hopkins adjusted clinical groups risk adjustment system. PLoS One 2019;14:e0221233.

9 Jvion. Jvion: readmission reduction with prescriptive analytics for preventable harm, 2017. Available: https://jvion.com/impact/ readmissions

10 Medial EarlySign. New Study Shows EarlySign's Machine Learning Algorithm Can Predict Which Cardiac Patients are at High-Risk Following Discharge. Cision PR Newswire, 2019. Available: https://www.prnewswire.com/il/news-releases/new-study-showsearlysigns-machine-learning-algorithm-can-predict-whichcardiac-patients-are-at-high-risk-following-discharge-300911407. $\mathrm{html}$

11 Dalkey N, Helmer O. An experimental application of the Delphi method to the use of experts. Manage Sci 1963;9:458-67.

12 Goodman CM. The Delphi technique: a critique. J Adv Nurs 1987;12:729-34.

13 Katrak P, Bialocerkowski AE, Massy-Westropp N, et al. A systematic review of the content of critical appraisal tools. BMC Med Res Methodol 2004:4:22.

14 J. Skulmoski G, T. Hartman F, Krahn J. The Delphi method for graduate research. JITE:Research 2007;6:001-21.

15 Anderson GF, Steinberg EP. Predicting Hospital readmissions in the Medicare population. Inquiry 1985;22:251-8.

16 Bottle A, Aylin P, Majeed A. Identifying patients at high risk of emergency hospital admissions: a logistic regression analysis. $J R$ Soc Med 2006;99:406-14.

17 Krumholz HM, Normand S-LT, Keenan PS. Hospital 30-day acute myocardial infarction readmission measure methodology, 2008.

18 Krumholz H, Normand S, Keenan P. Hospital 30-day heart failure readmission measure methodology, 2008.

19 Krumholz HM, Normand S-LT, Keenan PS. Hospital 30-day pneumonia readmission measure methodology, 2008.

20 Halfon P, Eggli Y, Prêtre-Rohrbach I, et al. Validation of the potentially avoidable Hospital readmission rate as a routine indicator of the quality of hospital care. Med Care 2006;44:972-81.

21 Hammill BG, Curtis LH, Fonarow GC, et al. Incremental value of clinical data beyond claims data in predicting 30-day outcomes after heart failure hospitalization. Circ Cardiovasc Qual Outcomes 2011;4:60-7.

22 Holloway JJ, Medendorp SV, Bromberg J. Risk factors for early readmission among Veterans. Health Serv Res 1990;25:213-37.

23 Holman C D'Arcy J, Preen DB, Baynham NJ, et al. A multipurpose comorbidity scoring system performed better than the Charlson index. J Clin Epidemiol 2005;58:1006-14.

24 Howell S, Coory M, Martin J, et al. Using routine inpatient data to identify patients at risk of hospital readmission. BMC Health Serv Res 2009;9:96.

25 Naessens JM, Leibson CL, Krishan I, et al. Contribution of a measure of disease complexity (complex) to prediction of outcome and charges among hospitalized patients. Mayo Clin Proc 1992;67:1140-9.

26 Philbin EF, DiSalvo TG. Prediction of hospital readmission for heart failure: development of a simple risk score based on administrative data. J Am Coll Cardiol 1999;33:1560-6.

27 Silverstein MD, Qin H, Mercer SQ, et al. Risk factors for 30day Hospital readmission in patients $\geq 65$ years of age. Proc 2008;21:363-72.

28 Thomas JW. Does risk-adjusted readmission rate provide valid information on hospital quality? Inquiry 1996;33:258-70 http://www. ncbi.nlm.nih.gov/pubmed/8883460

29 Amarasingham R, Moore BJ, Tabak YP, et al. An automated model to identify heart failure patients at risk for 30 -day readmission or death using electronic medical record data. Med Care 2010;48:981-8. 
30 Billings J, Mijanovich T. Improving the management of care for highcost Medicaid patients. Health Aff 2007;26:1643-54.

31 Billings J, Dixon J, Mijanovich T, et al. Case finding for patients at risk of readmission to hospital: development of algorithm to identify high risk patients. BMJ 2006;333:327.

32 Coleman EA, Min S-joon, Chomiak A, et al. Posthospital care transitions: patterns, complications, and risk identification. Health Serv Res 2004;39:1449-65.

33 Krumholz HM, Chen YT, Wang Y, et al. Predictors of readmission among elderly survivors of admission with heart failure. Am Heart $J$ 2000;139:72-7.

34 Morrissey EF, McElnay JC, Scott M, et al. Influence of drugs, demographics and medical history on hospital readmission of elderly patients. Clin Drug Investig 2003;23:119-28.

35 Smith DM, Norton JA, McDonald CJ. Nonelective readmissions of medical patients. J Chronic Dis 1985;38:213-24.

36 Smith DM, Katz BP, Huster GA, et al. Risk factors for nonelective Hospital readmissions. J Gen Intern Med 1996;11:762-4 http:// www.ncbi.nlm.nih.gov/pubmed/9016426

37 van Walraven C, Dhalla IA, Bell C, et al. Derivation and validation of an index to predict early death or unplanned readmission after discharge from hospital to the community. CMAJ 2010;182:551-7.

38 Burns R, Nichols LO. Factors predicting readmission of older general medicine patients. J Gen Intern Med 1991;6:389-93.

39 Evans RL, Hendricks RD, Lawrence KV, et al. Identifying factors associated with health care use: a hospital-based risk screening index. Soc Sci Med 1988;27:947-54.

40 Hasan O, Meltzer DO, Shaykevich SA, et al. Hospital readmission in general medicine patients: a prediction model. J Gen Intern Med 2010;25:211-9.

41 Boult C, Dowd B, McCaffrey D, et al. Screening elders for risk of hospital admission. J Am Geriatr Soc 1993;41:811-7.

42 Billings J, Blunt I, Steventon A, et al. Development of a predictive model to identify inpatients at risk of re-admission within 30 days of discharge (PARR-30). BMJ Open 2012;2:e001667-10.

43 Choudhry SA, Li J, Davis D, et al. A public-private partnership develops and externally validates a 30-day Hospital readmission risk prediction model. Online J Public Health Inform 2013;5:219.

44 Cotter PE, Bhalla VK, Wallis SJ, et al. Predicting readmissions: poor performance of the lace index in an older UK population. Age Ageing 2012;41:784-9.

45 Donzé J, Aujesky D, Williams D, et al. Potentially avoidable 30-day Hospital readmissions in medical patients: derivation and validation of a prediction model. JAMA Intern Med 2013;173:632-8.

46 Hebert C, Shivade C, Foraker R, et al. Diagnosis-specific readmission risk prediction using electronic health data: a retrospective cohort study. BMC Med Inform Decis Mak 2014;14:65

47 Escobar GJ, Ragins A, Scheirer P, et al. Nonelective rehospitalizations and Postdischarge mortality: predictive models suitable for use in real time. Med Care 2015;53:916-23.

48 Yu S, Farooq F, van Esbroeck A, et al. Predicting readmission risk with institution-specific prediction models. Artif Intell Med 2015;65:89-96.

49 Baillie CA, VanZandbergen C, Tait G, et al. The readmission risk flag: using the electronic health record to automatically identify patients at risk for 30-day readmission. J Hosp Med 2013;8:689-95.

50 Gildersleeve R, Cooper P. Development of an automated, real time surveillance tool for predicting readmissions at a community hospital. Appl Clin Inform 2013;4:153-69.

51 Kruse RL, Hays HD, Madsen RW. Risk factors for all-cause Hospital readmission within 30 days of hospital discharge. JCOM 2013:20:203-14.

52 Richmond DM. Socioeconomic predictors of 30-day Hospital readmission of elderly patients with initial discharge destination of home health care 2013.

53 Shulan M, Gao K, Moore CD. Predicting 30-day all-cause Hospital readmissions. Health Care Manag Sci 2013;16:167-75.

54 Lee EW. Selecting the best prediction model for readmission. J Prev Med Public Health 2012;45:259-66.

55 van Walraven C, Wong J, Forster AJ. Derivation and validation of a diagnostic score based on case-mix groups to predict 30-day death or urgent readmission. Open Med 2012;6:e90-100.

56 van Walraven C, Wong J, Forster AJ. LACE+ index: extension of a validated index to predict early death or urgent readmission after hospital discharge using administrative data. Open Med 2012;6:e80-90.

57 lannuzzi JC, Chandra A, Kelly KN, et al. Risk score for unplanned vascular readmissions. J Vasc Surg 2014;59:1340-7.

58 Keyhani S, Myers LJ, Cheng E, et al. Effect of clinical and social risk factors on hospital profiling for stroke readmission: a cohort study. Ann Intern Med 2014;161:775-84.
59 Rana S, Tran T, Luo W, et al. Predicting unplanned readmission after myocardial infarction from routinely collected administrative hospital data. Aust Health Rev 2014;38:377-82.

60 Shahian DM, He X, O'Brien SM, et al. Development of a clinical registry-based 30-day readmission measure for coronary artery bypass grafting surgery. Circulation 2014;130:399-409.

61 Shams I, Ajorlou S, Yang K. A predictive analytics approach to reducing 30-day avoidable readmissions among patients with heart failure, acute myocardial infarction, pneumonia, or COPD. Health Care Manag Sci 2015;18:19-34.

62 Sharif R, Parekh TM, Pierson KS, et al. Predictors of early readmission among patients 40 to 64 years of age hospitalized for chronic obstructive pulmonary disease. Ann Am Thorac Soc 2014;11:685-94.

63 Lucas DJ, Haider A, Haut E, et al. Assessing readmission after general, vascular, and thoracic surgery using ACS-NSQIP. Ann Surg 2013;258:430-9.

64 Wallmann R, Llorca J, Gómez-Acebo I, et al. Prediction of 30-day cardiac-related-emergency-readmissions using simple administrative hospital data. Int J Cardiol 2013;164:193-200.

65 Wasfy JH, Rosenfield K, Zelevinsky K, et al. A prediction model to identify patients at high risk for 30-day readmission after percutaneous coronary intervention. Circ Cardiovasc Qual Outcomes 2013;6:429-35.

66 Krumholz HM, Lin Z, Drye EE, et al. An administrative claims measure suitable for profiling Hospital performance based on 30-day all-cause readmission rates among patients with acute myocardial infarction. Circ Cardiovasc Qual Outcomes 2011;4:243-52.

67 Betihavas V, Frost SA, Newton PJ, et al. An absolute risk prediction model to determine unplanned cardiovascular readmissions for adults with chronic heart failure. Heart Lung Circ 2015;24:1068-73.

$68 \mathrm{Di}$ Tano G, De Maria R, Gonzini L, et al. The 30-day metric in acute heart failure revisited: data from IN-HF outcome, an Italian nationwide cardiology registry. Eur J Heart Fail 2015;17:1032-41.

69 Huynh QL, Saito M, Blizzard CL, et al. Roles of nonclinical and clinical data in prediction of 30-day rehospitalization or death among heart failure patients. J Card Fail 2015;21:374-81.

70 Raposeiras-Roubín S, Abu-Assi E, Cambeiro-González C, et al. Mortality and cardiovascular morbidity within 30 days of discharge following acute coronary syndrome in a contemporary European cohort of patients: how can early risk prediction be improved? the six-month grace risk score. Rev Port Cardiol 2015;34:383-91.

71 Fleming LM, Gavin M, Piatkowski G, et al. Derivation and validation of a 30-day heart failure readmission model. Am J Cardiol 2014;114:1379-82

72 Eapen ZJ, Liang L, Fonarow GC, et al. Validated, electronic health record deployable prediction models for assessing patient risk of 30-day rehospitalization and mortality in older heart failure patients. JACC Heart Fail 2013;1:245-51.

73 Zai AH, Ronquillo JG, Nieves R, et al. Assessing Hospital readmission risk factors in heart failure patients enrolled in a telemonitoring program. Int J Telemed Appl 2013;2013:305819.

74 Au AG, McAlister FA, Bakal JA, et al. Predicting the risk of unplanned readmission or death within 30 days of discharge after a heart failure hospitalization. Am Heart J 2012;164:365-72.

75 Watson AJ, O'Rourke J, Jethwani K, et al. Linking electronic health record-extracted psychosocial data in real-time to risk of readmission for heart failure. Psychosomatics 2011;52:319-27.

76 Mather JF, Fortunato GJ, Ash JL, et al. Prediction of pneumonia 30-day readmissions: a single-center attempt to increase model performance. Respir Care 2014:59:199-208.

77 Lindenauer PK, Normand S-LT, Drye EE, et al. Development, validation, and results of a measure of 30-day readmission following hospitalization for pneumonia. J Hosp Med 2011;6:142-50.

78 Shadmi E, Flaks-Manov N, Hoshen M, et al. Predicting 30-day readmissions with preadmission electronic health record data. Med Care 2015:53:283-9.

79 Tsui E, Au SY, Wong CP, et al. Development of an automated mode to predict the risk of elderly emergency medical admissions within a month following an index Hospital visit: a Hong Kong experience. Health Informatics J 2015;21:46-56.

80 Donzé J, Lipsitz S, Schnipper JL. Risk factors for potentially avoidable readmissions due to end-of-life care issues. $J$ Hosp Med 2014;9:310-4

$81 \mathrm{He}$ D, Mathews SC, Kalloo AN, et al. Mining high-dimensional administrative claims data to predict early hospital readmissions. $J$ Am Med Inform Assoc 2014;21:272-9.

82 Taha M, Pal A, Mahnken JD, et al. Derivation and validation of a formula to estimate risk for 30-day readmission in medical patients. Int J Qual Health Care 2014;26:271-7. 
83 Zapatero A, Barba R, Marco J, et al. Predictive model of readmission to internal medicine wards. Eur J Intern Med 2012;23:451-6.

84 Singal AG, Rahimi RS, Clark C, et al. An automated model using electronic medical record data identifies patients with cirrhosis at high risk for readmission. Clin Gastroenterol Hepatol 2013:11:1335-41.

85 Volk ML, Tocco RS, Bazick J, et al. Hospital readmissions among patients with decompensated cirrhosis. Am J Gastroenterol 2012;107:247-52.

86 Perkins RM, Rahman A, Bucaloiu ID, et al. Readmission after hospitalization for heart failure among patients with chronic kidney disease: a prediction model. Clin Nephrol 2013;80:433-40.

87 Nijhawan AE, Clark C, Kaplan R, et al. An electronic medical record-based model to predict 30-day risk of readmission and death among HIV-infected inpatients. J Acquir Immune Defic Syndr 2012;61:349-58.

88 Whitlock TL, Tignor A, Webster EM, et al. A scoring system to predict readmission of patients with acute pancreatitis to the hospital within thirty days of discharge. Clin Gastroenterol Hepatol 2011;9:175-80.

89 Taber DJ, Palanisamy AP, Srinivas TR, et al. Inclusion of dynamic clinical data improves the predictive performance of a 30-day readmission risk model in kidney transplantation. Transplantation 2015;99:324-30.

90 Lawson EH, Hall BL, Louie R, et al. Identification of modifiable factors for reducing readmission after colectomy: a national analysis. Surgery 2014;155:754-66.

91 lannuzzi JC, Fleming FJ, Kelly KN, et al. Risk scoring can predict readmission after endocrine surgery. Surgery 2014;156:1432-8. discussion 1438-40.

92 Mesko NW, Bachmann KR, Kovacevic D, et al. Thirty-day readmission following total hip and knee arthroplasty - a preliminary single institution predictive model. J Arthroplasty 2014;29:1532-8.

93 Moore L, Stelfox HT, Turgeon AF, et al. Derivation and validation of a quality indicator for 30-day unplanned Hospital readmission to evaluate trauma care. J Trauma Acute Care Surg 2014;76:1310-6.

94 Graboyes EM, Liou T-N, Kallogjeri D, et al. Risk factors for unplanned Hospital readmission in otolaryngology patients. Otolaryngol Head Neck Surg 2013;149:562-71.

95 Vigod SN, Kurdyak PA, Seitz D, et al. READMIT: a clinical risk index to predict 30-day readmission after discharge from acute psychiatric units. J Psychiatr Res 2015;61:205-13.

96 Kuhn M, Johnson K. Applied predictive modeling 2013.

97 Wiens J, Saria S, Sendak M, et al. Do no harm: a roadmap for responsible machine learning for health care. Nat Med 2019;25:1337-40.

98 Ancker JS, Kim M-H, Zhang Y, et al. The potential value of social determinants of health in predicting health outcomes. J Am Med Inform Assoc 2018:25:1109-10.
99 National Quality Forum. Measure evaluation criteria, 2012. Available: http://www.qualityforum.org/Measuring_Performance/Submitting_ Standards/Measure_Evaluation_Criteria.aspx

100 Lundberg SM, Lee S-I. A unified approach to interpreting mode predictions. Adv Neural Inf Process Syst 2017:4765-74.

101 Kripalani S, Theobald CN, Anctil B, et al. Reducing Hospital readmission rates: current strategies and future directions. Annu Rev Med 2014;65:471-85.

102 Schneider EB, Hyder O, Brooke BS, et al. Patient readmission and mortality after colorectal surgery for colon cancer: impact of length of stay relative to other clinical factors. J Am Coll Surg 2012;214:390-8.

103 Centers for Medicare \& Medicaid Services. CMS.gov: Hospital readmissions reduction program (HRRP), 2013. Available: https:// www.cms.gov/medicare/medicare-fee-for-service-payment/ acuteinpatientpps/readmissions-reduction-program.html

104 Goldfield NI, McCullough EC, Hughes JS, et al. Identifying potentially preventable readmissions. Health Care Financ Rev 2008;30:75-91.

105 Walsh C, Hripcsak G. The effects of data sources, cohort selection, and outcome definition on a predictive model of risk of thirty-day Hospital readmissions. J Biomed Inform 2014;52:418-26.

106 Lipton ZC. The Mythos of model Interpretability, 2016.

107 Austin PC, Brunner LJ. Inflation of the type I error rate when a continuous confounding variable is categorized in logistic regression analyses. Stat Med 2004;23:1159-78.

108 Steyerberg EW, Harrell FE. Prediction models need appropriate internal, internal-external, and external validation. J Clin Epidemiol 2016;69:245-7.

109 Austin PC, Steyerberg EW. Events per variable (EPV) and the relative performance of different strategies for estimating the outof-sample validity of logistic regression models. Stat Methods Med Res 2017;26:796-808.

110 Molinaro AM, Simon R, Pfeiffer RM. Prediction error estimation: a comparison of resampling methods. Bioinformatics 2005;21:3301-7.

111 Harrell FE. Regression Modeling Strategies: With Applications to Linear Models, Logistic and Ordinal Regression, and Survival Analysis. 2nd Editio. Cham: Springer International Publishing, 2015.

112 Steyerberg EW, Vickers AJ, Cook NR, et al. Assessing the performance of prediction models: a framework for traditional and novel measures. Epidemiology 2010;21:128-38.

113 Crowson CS, Atkinson EJ, Therneau TM. Assessing calibration of prognostic risk scores. Stat Methods Med Res 2016;25:1692-706.

114 Davis SE, Greevy RA, Fonnesbeck C, et al. A nonparametric updating method to correct clinical prediction model drift. J Am Med Inform Assoc 2019;26:1448-57. 